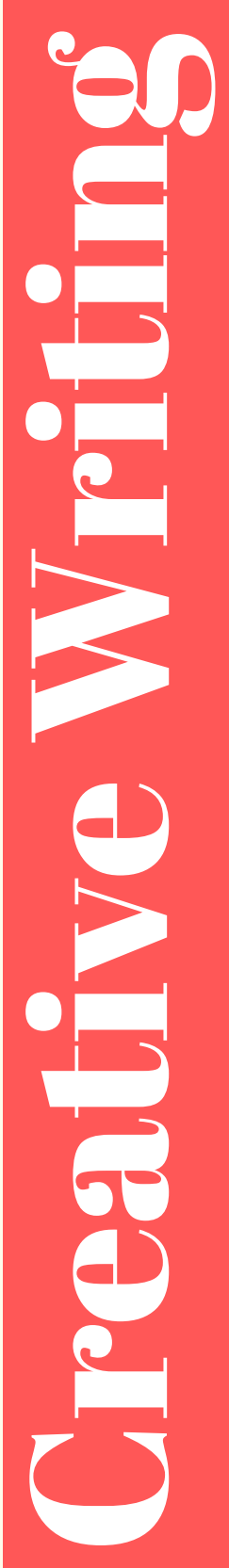




\section{Everything Changes}

\section{Lina María Calixto}

Everything changes when the sun no longer heats, when the wind no longer whistles.

Love changes, songs change, and the weather changes.

Everything changes when the fire goes out, when the rain is thick, when the clouds get dark.

The past changes, the madness changes, and the people's essence changes.

Everything changes when memories do not hurt, when cold hands do not feel each other anymore.

The art of smiling changes, reasons change, and opportunities change.

Everything changes when lyrics are not play anymore, and when the mind seems empty.

Dreams change, roots change, the meaning of words changes.

Everything changes when the pencil does not stain, when the sheets are torn.

Inspiration changes, colors change, and imagination changes.

Everything changes when the rhythm is slow, when energies do not fit each other.

Sighs change, bodies change, and thoughts change.

Consequently, everything changes, and it is okay.

Every day, something changes, and ...

it changes for good. 